Pesq. Vet. Bras. 35(2):193-198, fevereiro 2015 DOI: 10.1590/S0100-736X2015000200017

\title{
Morfologia e morfometria da traqueia da preguiça (Bradypus variegatus): conhecimentos para procedimentos de emergência ${ }^{1}$
}

\author{
Thamirys A.S. Faro ${ }^{2}$, Ana Rita de Lima², Antonio C. Messias ${ }^{3}$, Rosa Cabral ${ }^{2}$, Elane G. \\ Giese $^{2}$, Edilson R. Matos ${ }^{2}$ eÉrika Branco ${ }^{2 *}$
}

\begin{abstract}
Faro T.A.S., Lima A.R., Messias A.C., Cabral R., Giese E.G., Matos E.R. \& Branco E. 2015. [Morphological and morphometric trachea sloth (Bradypus variegatus): knowledge for emergency procedures.] Morfologia e morfometria da traqueia da preguiça (Bradypus variegatus): conhecimentos para procedimentos de emergência. Pesquisa Veterinária Brasileira 35(2):193-198. Setor de Anatomia Descritiva dos Animais Domésticos e Selvagens, Instituto de Saúde e Produção Animal, Faculdade de Medicina Veterinária, Universidade Federal Rural da Amazônia, Campus de Belém, PA 66077-530, Brazil. E-mail: ebranco.ufra@gmail.com

As the sloth (Bradypus variegatus) is a little studied species, especially from a morphological point of view, this research aimed to define the anatomy of its trachea. The information would facilitate the selection of a proper endotracheal tube, laryngeal mask or tracheostomy tube for anesthesia and emergency procedures, since it appeared to have a special morphology. Eleven young animals of different ages were investigated, four males and seven females, obtained from the Emilio Goeldi Museum and donated to UFRA. The specimens were infused intramuscularly with $10 \%$ aqueous formaldehyde for preservation and were later dissected at the cervico-thoracic level, by mesoscopia, exposing the area from the larynx to the right and left primary bronchi at the hilum. The tracheae were divided into five regions (cervical, first flexure, second flexure, third flexure, and carina) for which length and width were measured, as well as the total tracheal length. Sharp windings were seen in the middle caudal portion, including the carina. The average tracheal length was $14.6 \mathrm{~cm}$. Microscopically, the trachea was made up of separate plates of hyaline cartilage forming each ring, lined with ciliated epithelium. Despite the trachea of the common sloth displaying the same lining pattern found in other animals, there are no reports in the literature of other species having a macroscopic morphology as described here, which leads us to suggest, where appropriate access to emergency ventilation, the practice of IOT and not tracheostomy.
\end{abstract}

INDEX TERMS: Anatomy, histology, trachea, sloth, Bradypus variegatus.

RESUMO.- Por ser uma espécie pouco estudada, principalmente do ponto de vista morfológico, objetivou-se conhecer a anatomia da traqueia da preguiça (Bradypus variegatus) a fim de fornecer informações para facilitar a eleição de

\footnotetext{
${ }^{1}$ Recebido em 22 de julho de 2014.

Aceito para publicação em 31 de janeiro de 2015.

${ }^{2}$ Laboratório de Pesquisa Morfológica Animal (LaPMA), Faculdade de Medicina Veterinária, Universidade Federal Rural da Amazônia (UFRA), Av. Presidente Tancredo Neves 2501, Belém, PA 66077-530, Brasil. *Autor para correspondência: ebranco.ufra@gmail.com

${ }^{3}$ Museu Paraense Emílio Goeldi, Av. Magalhães Barata 376, Belém, PA 66040-170.
}

tubo endotraqueal adequado, máscara laríngea ou cânula de traqueostomia para anestesia e procedimentos de emergência, uma vez que a mesma revelou-se possuidora de uma morfologia especial. Foram investigados 11 animais jovens de idades diferentes, sendo quatro machos e sete fêmeas, provenientes do Museu Emilio Goeldi e doados a UFRA. Os exemplares foram perfundidos via intramuscular com solução aquosa de formol a 10\% para fins de conservação e posteriormente foram dissecados em nível cervico-torácico, por meio de mesoscopia, expondo-se desde a laringe até os brônquios principais direito e esquerdo ao nível do hilo pulmonar. As traqueias foram divididas em cinco regiões (região 
cervical, região da primeira flexura, região da segunda flexura, região da terceira flexura e região da carina) as quais foram mensuradas altura e largura, assim como o comprimento traqueal total e parte do material foi submetido à técnicas de rotina histológica. Macroscopicamente, destacou-se a presença de sinuosidades acentuadas em porção médio caudal, contemplando a carina. 0 comprimento médio traqueal foi de $14,6 \mathrm{~cm}$. Microscopicamente, a traqueia era constituída por placas separadas de cartilagem hialina constituindo cada anel, sendo revestido por epitélio estratificado ciliado. Apesar da traqueia da preguiça comum apresentar revestimento padrão encontrado na traqueia de outros animais, na literatura não há registros de outras espécies que tenham morfologia macroscópica nas condições descritas, o que nos leva a sugerir, quando necessário acesso para ventilação de emergência, a prática da IOT e não a de traqueostomia.

TERMOS DE INDEXAÇÃO: Anatomia, histologia, traqueia, preguiça comum, Bradypus variegatus.

\section{INTRODUÇÃO}

Pertencendo à classe Mammalia, ordem Xenarthra e família Bradypodydae, a preguiça (Bradypus variegatus) é encontrada tanto nas florestas secas como nas verdejantes e até em árvores isoladas em pastos, vivendo nas áreas altas sendo de difícil visualização. Está presente em florestas primárias e secundárias, mas também pode ser vista em áreas com vegetação baixa. Diversas vezes esses animais são capturados nas proximidades de estradas e colocados em jardins e parques nas cidades, onde podem sobreviver. A distribuição geográfica de Bradypus variegatus se dá na América Central e do Sul, Sul de Honduras, oeste dos Andes até o sul do Equador, leste dos Andes, do norte da Venezuela e Colômbia até a Bolívia e norte da Argentina, exceto na área leste do Rio Negro e norte do Amazonas, em até aproximadamente $1.100 \mathrm{~m}$ de elevação (Cáuper et al. 2006).

A maior característica para determinação da espécie é a presença de três dedos e pescoço marrom (Amorin et al. 2003), possuindo também a cabeça pequena e redonda; orelhas não visíveis, face em tons de branco ou marrom, muitas vezes com faixas escuras na testa, faixa preta em torno dos olhos, que se estende até a parte de trás das orelhas, além de um leve "sorriso" e um focinho preto. Possui uma dieta baseada em folhas de várias espécies de plantas. Geralmente se pendura com suas garras de cabeça para baixo ou se senta em uma bifurcação nos galhos da árvore com a cabeça entre os membros torácicos. Preferindo árvores de copa alta para poder se expor ao sol, também podem ser vistos em arbustos expostos. No solo, são praticamente incapazes de se locomover, já nos rios, podem nadar, e podem ser vistas atravessando os mesmos (Cáuper et al. 2006).

Quanto à traqueia, órgão alvo deste estudo, é constituída de tecido conjuntivo tubular flexível, que se estende da cartilagem cricóide da laringe até a carina, apresentando uma porção cervical e uma torácica. A traqueia inteira deve ser flexível para permitir o movimento da cabeça, do pescoço e da laringe. Essa flexibilidade é possível devido à cartilagem ser formada por anéis individuais conectados por ligamentos fibroelásticos (Ettinger \& Feldman 2004).
Em termos histológicos, a traqueia é composta por três camadas: mucosa, submucosa e adventícia. A mucosa é constituída pelo epitélio respiratório, lâmina própria e lâmina elástica. 0 epitélio respiratório ou de revestimento, é do tipo pseudo estratificado cilíndrico ciliado, e apresenta vários tipos celulares como, por exemplo, as células caliciformes (Ross \& Pawlina 2012).

Neste cenário, este estudo propõe a investigação morfológica e morfométrica da traqueia da Bradypus variegatus, pois, pouco se sabe sobre os aspectos macro e microscópicos de diversos órgãos dessa espécie, visando com isso auxiliar condutas terapêuticas e anestésicas, lembrando que com relativa frequência estes animais são vítimas de traumas causados por atropelamentos a beira de estradas e acidentes de captura, necessitando muitas vezes de procedimentos invasivos como intubação orotraqueal (IOT) ou traqueostomia para manutenção da vida e tratamento médico veterinário de moderada complexidade, como a utilização de fármacos para a contenção e manutenção anestésica (Gilmore et al. 2000), sugerindo possíveis sondas endotraqueais.

\section{MATERIAL E MÉTODOS}

Todos os procedimentos realizados nesse estudo estão de acordo com a liberação do IBAMA (SISBIO), sob no2 23401-2.

Foram utilizados onze animais jovens, sendo três machos com idade aproximada variando entre cinco e sete meses e sete fêmeas com idade variando de aproximadamente cinco meses e três semanas, e um animal adulto do sexo masculino, os quais foram a óbito por causas naturais que não comprometeram o sistema respiratório, e doados ao Laboratório de Pesquisa Morfológica Animal (LaPMA) da Universidade Federal Rural da Amazônia (UFRA).

Os exemplares foram perfundidos via intramuscular com solução aquosa de formol a $10 \%$ para fixação e conservação, e mantidos em cubas com a mesma solução fixadora por sete dias. Posteriormente os mesmos foram dissecados em nível cervico-torácico, com abordagem ventral, expondo-se desde a laringe até os brônquios principais direito e esquerdo à altura do hilo pulmonar.

0 material foi dissecado por mesoscopia, com auxílio de material básico de dissecação, procedendo-se em seguida a contagem do número de anéis cartilaginosos traqueais de cada animal. Baseando-se nesta informação, a traqueia foi dividida em cinco regiões, designadas como região cervical, região da primeira flexura, região da segunda flexura, região da terceira flexura e região da carina traqueal.

O comprimento da traqueia foi mensurado do primeiro ao último anel no sentido crânio-caudal, com auxílio de fio de nylon, que acompanhou toda a extensão e flexuras do órgão e em seguida foi estendido sobre fita métrica revelando as medidas aferidas. Por fim, foram tomados os diâmetros correspondentes à altura (dorso-ventral) e largura (latero-lateral) das cinco regiões com auxilio de paquímetro digital (Western DC-6). A análise estatística foi feita por meio do programa InStat.

Para análise microscópica foram retirados fragmentos das cinco porções da traqueia e fixados em formaldeído 10\%. Após a fixação, o material foi desidratado em série etanóica (de 70 a $100 \%$ ), clarificados em xilol e incluídos em parafina para posterior microtomia. Os cortes foram corados com Hematoxilina e Eosina e o material foi analisado, mapeado e fotografado através de microscópio Leica ICC50 com captura digital de imagens.

Toda nomenclatura adotada foi baseada na Nomenclatura Atômica Veterinária (International Committee onn Veterinary Gross Anatomical Nomenclature 2012). 


\section{RESULTADOS}

A traqueia de Bradypus variegatus apresentou sinuosidades em porção médio caudal, contemplando a carina traqueal, sendo este fato, uma exclusividade desta espécie. Foram calculadas as médias dos comprimentos traqueais e dos números de anéis (Quadro 1).

Em B. variegatus obteve-se uma média de 100 anéis incompletos, variando desta forma o comprimento da traqueia em relação ao número de anéis. No primeiro grupo (machos), o comprimento traqueal foi de $18,4 \mathrm{~cm}$. 0 número total de anéis contabilizados do primeiro ao último foi de 106 anéis. Já no segundo grupo (fêmeas), o comprimento traqueal foi de $12,4 \mathrm{~cm}$ e o número total de anéis, 101.

Em relação às sinuosidades, em todos os animais, as primeiras surgiram a partir da porção inicial do lobo pulmonar esquerdo (Fig.1A,B). Já no macho de sete meses, as sinuosidades originaram-se na porção inicial dos dois lobos (Fig.1B).

A altura e a largura das cinco regiões de cada animal foram mensuradas e comparadas mostrando resultados diferentes entre si, sendo calculadas as médias (Quadro 2).

Foram encontradas variações nas sinuosidades traqueais, de forma que a maioria dos animais apresentou duas sinuosidades, porém, em uma fêmea de cinco meses e em um macho de sete meses, encontrou-se três sinuosidades traqueais (Quadro 2).

Ao analisar os valores médios obtidos para a altura da região cervical, primeira, segunda, terceira flexuras e região da carina da traqueia de $B$. variegatus, pelo teste de Tukey e nível de significância $<0,05$, foram obtidas diferenças estatisticamente significantes entre:

Quadro 1. Comprimento traqueal e número total de anéis traqueais de Bradypus variegatus machos e fêmeas

\begin{tabular}{lccc}
\hline Idade do animal & Sexo & $\begin{array}{c}\text { Comprimento } \\
\text { traqueal }(\mathrm{cm})\end{array}$ & Anéis traqueais \\
\hline Cinco meses & $\mathrm{M}$ & 16 & 80 \\
Cinco meses & $\mathrm{M}$ & 12 & 135 \\
Sete meses* & $\mathrm{F}$ & 19 & 81 \\
Três semanas & $\mathrm{F}$ & 12,5 & 78 \\
Três semanas & $\mathrm{F}$ & 11,6 & 88 \\
Três semanas & $\mathrm{F}$ & 11 & 130 \\
Cinco meses & $\mathrm{F}$ & 12,5 & 89 \\
Cinco meses & $\mathrm{F}$ & 14 & 101 \\
Cinco meses* & $\mathrm{F}$ & 16 & 131 \\
Cinco meses & $\mathrm{F}$ & 9,5 & 93 \\
Média & & 13,41 & 100,6 \\
Desvio Padrão & & 2,84 & 22,7
\end{tabular}

*Animais que apresentaram três sinuosidades.
Alturas da região cervical dos animais de 3 semanas e da região cervical dos animais com 5 meses;

Alturas da região cervical e da região carina dos animais de 5 meses, e primeira e terceira flexuras dos animais de 7 meses;

Alturas da primeira flexura dos animais de 3 semanas, da região cervical dos animais de 5 meses e da região da carina, primeira, segunda e terceira flexuras dos animais de 7 meses;

Alturas da segunda flexura dos animais de 3 semanas, da região cervical dos animais de 5 meses, e da primeira, segunda e terceira flexuras dos animais de 7 meses;

Alturas da região da carina dos animais de 3 semanas, da região cervical dos animais de 5 meses, e da região cervical, região da carina, primeira, segunda e terceira flexuras dos animais de 7 meses;

Alturas da região cervical dos animais de 5 meses, da região da carina, primeira, segunda, terceira flexuras dos animais de 5 meses, e da região cervical, região da carina, primeira, segunda e terceira flexura dos animais de 7 meses;

Alturas da primeira flexura dos animais com 5 meses e da primeira, segunda e terceira flexuras dos animais com 7 meses;

Alturas da segunda flexura dos animais com 5 meses e da região da carina e primeira, segunda e terceira flexuras dos animais com 7 meses;

Alturas da região da carina dos animais com 5 meses e da primeira, segunda e terceira flexuras dos animais com 7 meses;

Alturas da terceira flexura dos animais com 5 meses e da primeira e terceira flexuras dos animais com 7 meses.

Ao analisar os valores médios obtidos para a largura da região cervical, primeira flexura, segunda flexura, terceira flexura e região da carina da traqueia de $B$. variegatus, pelo teste de Tukey e nível de significância $<0,05$ foram obtidas diferenças estatisticamente significantes entre:

Larguras da região cervical dos animais de três semanas, da região da região da carina e terceira flexura dos animais de 5 meses;

Larguras da região cervical dos animais de três semanas, da região cervical e da região da carina, primeira e segunda flexuras dos animais de 7 meses;

Larguras da primeira flexura dos animais de três semanas, da região cervical, da região da carina, primeira e segunda flexuras dos animais de 7 meses;

Larguras da segunda flexura dos animais de três semanas, da região da carina e terceira flexura dos animais de 5 meses;

Quadro 2. Alturas e larguras das cinco regiões da traqueia da Bradypus variegatus de acordo com a idade de cada animal e médias em cm

\begin{tabular}{|c|c|c|c|c|c|c|c|c|c|c|c|c|c|}
\hline \multirow{2}{*}{$\begin{array}{l}\text { Altura/ } \\
\text { Largura }\end{array}$} & \multicolumn{11}{|c|}{ Idade dos animais } & \multirow{2}{*}{$\begin{array}{c}\text { Média } \\
\text { da al- } \\
\text { tura }\end{array}$} & \multirow{2}{*}{$\begin{array}{c}\text { Média } \\
\text { da lar- } \\
\text { gura }\end{array}$} \\
\hline & & Três semanas & & & Cinco meses $^{1}$ & & & Cinco meses $^{2}$ & & Sete meses $^{2}$ & Adulto $^{1}$ & & \\
\hline Região cervical & $0,32 / 0,36$ & $0,28 / 0,33$ & $0,30 / 0,35$ & $0,39 / 0,44$ & $0,51 / 0,56$ & $0,38 / 0,43$ & $0,38 / 0,48$ & $0,36 / 0,43$ & $0,43 / 0,43$ & $0,50 / 0,55$ & $0,71 / 0,89$ & 0,41 & 0,47 \\
\hline Segunda flexura & $0,33 / 0,42$ & $0,24 / 0,31$ & $0,38 / 0,41$ & $0,43 / 0,50$ & $0,36 / 0,39$ & $0,32 / 0,43$ & $0,36 / 0,43$ & $0,24 / 0,35$ & $0,56 / 0,45$ & $0,50 / 0,63$ & $1,01 / 0,92$ & 0,43 & 0,47 \\
\hline Terceira flexura & - & - & - & - & - & - & $0,45 / 0,50$ & - & - & $0,43 / 0,75$ & - & 0,44 & 0,63 \\
\hline Região da carina & $0,40 / 0,44$ & $0,27 / 0,28$ & $0,27 / 0,33$ & $0,52 / 0,67$ & $0,51 / 0,34$ & $0,44 / 0,52$ & $0,44 / 0,44$ & $0,33 / 0,48$ & $0,38 / 0,30$ & $0,66 / 0,62$ & $0,47 / 1,06$ & 0,42 & 0,50 \\
\hline
\end{tabular}



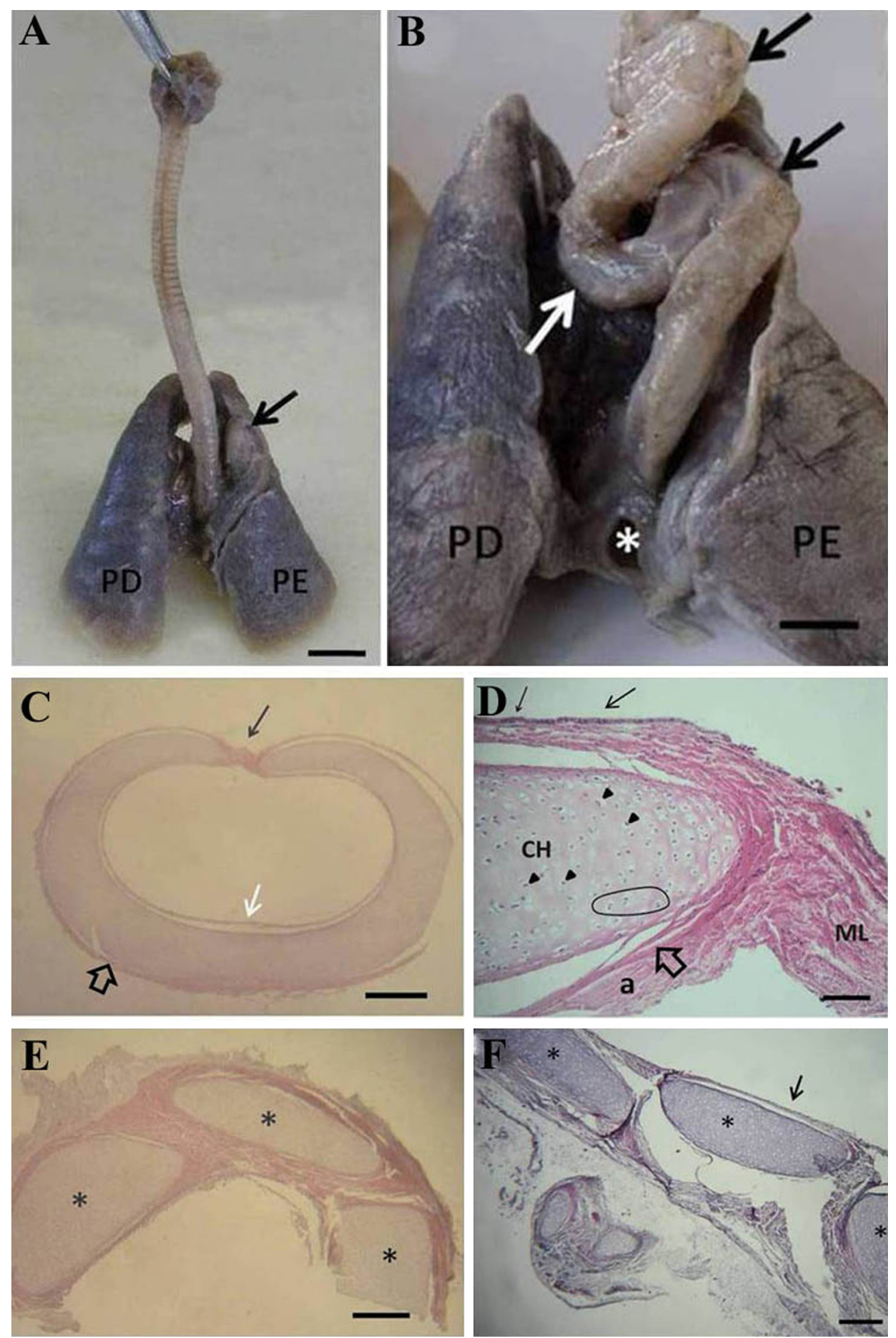

Fig.1. Traqueia de Bradypus variegatus. (A,B) Terço final com parte das sinuosidades aparentes (setas). Observar detalhe das sinuosidades do terço final da traqueia dispostas de forma cranial, médio e caudal (setas), desembocando junto à carina traqueal $(*)$. Lobo pulmonar direito (PD), Lobo pulmonar esquerdo (PE). Barra $=1 \mathrm{~cm}$. (C) Corte transversal na região cervical. Observar a não segmentação da cartilagem com suas extremidades sendo ligadas por prega de músculo liso (seta preta). Notar epitélio de revestimento (epitélio simples pseudo estratificado) (seta branca) e pericôndrio (seta vazada). (D) Corte histológico do anel cartilaginoso da traquéia de Bradipus variegatus. Cartilagem hialina limitada pelo pericôndrio (p), onde logo abaixo se visualiza condroblastos (cb) e condrócitos (cc) imersos na matriz cartilaginosa hialina (mc). Mais internamente, observa-se a presença de condrócitos formando grupos isógenos (cabeça de seta). (E) Corte mediano na região da primeira flexura (cranial). Observar a segmentação da cartilagem $\left({ }^{*}\right)$. (F) Corte mediano na região da carina traqueal. Observar a segmentação da cartilagem $\left({ }^{*}\right.$ ), assim como em C. (C, E e F) Coloração em HE, barra $=200 \mu \mathrm{m}$. (D) Coloração pelo Tricrômico de Gomory. Barra de escala: $20 \mathrm{um}$ 
Larguras da segunda flexura dos animais de três semanas, da região cervical, da região da carina e primeira e segunda flexuras dos animais de 7 meses;

Larguras da região da carina dos animais de três semanas, da região da carina e terceira flexura dos animais de 5 meses;

Larguras da região da carina dos animais de três semanas, da região cervical, da região da carina, primeira e segunda flexuras dos animais de 7 meses;

Larguras da região cervical dos animais de 5 meses e da região da carina dos animais de 7 meses;

Larguras da primeira flexura dos animais de 5 meses e da região da carina dos animais de 7 meses;

Larguras da segunda flexura dos animais de 5 meses, da região cervical, da região da carina e da segunda flexura dos animais de 7 meses;

Larguras da região da carina dos animais de 5 meses e da região da carina dos animais de 7 meses;

Larguras da terceira flexura dos animais de 5 meses e da região da carina dos animais de 7 meses;

Larguras da região cervical dos animais de 7 meses e da região da carina dos animais de 7 meses;

Larguras da primeira flexura dos animais de 7 meses e da região da carina dos animais de 7 meses;

Larguras da segunda flexura dos animais de 7 meses e da região da carina dos animais de 7 meses;

Larguras da terceira flexura dos animais de 7 meses e da região da carina dos animais de 7 meses.

Microscopicamente, as cartilagens da primeira, segunda, terceira flexura e da região da carina (Fig.1F) apresentaram segmentação com formações de placas de cartilagem hialina, todavia não registrou-se variação microscópica entres elas.

Além disso, observou-se as estruturas clássicas da traqueia, tais como o epitélio de revestimento (epitélio cilíndrico pseudo-estratificado ciliado) e grupos isogênicos de condrócitos na matriz da cartilagem hialina, pericôndrio e camada adventícia (Fig.1C-F).

\section{DISCUSSÃO}

A forma dos anéis traqueais é diferente para cada espécie animal (König \& Liebich 2004). Bradypus variegatus apresentou cartilagens com a forma de "C" somente na porção cervical e parte da torácica, no qual, as extremidades desses semi-anéis eram ligadas por um delicado feixe de músculo liso (Fig. 1C), assemelhando-se ao modelo canino e dos humanos, como descreveu Xavier et al. (2008), bem como Saimiri sciureus (Pinheiro et al. 2012), Lemuroidea (Straus 1931) e Siphonops annulatus (Kuehne \& Junqueira 2000). Ao contrário de gansos em que a traqueia apresentou anéis completos (Santos et al. 2008).

O comprimento da traqueia e o número de anéis cartilaginosos variam tanto dentre o grupo de uma única espécie quanto de uma espécie para outra. No equino e nos ruminantes há de 48 a 60 anéis; no cão, de 42 a 46 anéis; no gato, de 38 a 46 anéis; e no suíno de 32 a 36 anéis (Getty 1986, Dyce et al. 2004, König \& Liebich 2004). Em B. variegatus obteve-se uma média de 100 anéis incompletos, variando desta forma o comprimento da traqueia em relação ao número de anéis. No primeiro grupo (machos), o comprimento traqueal foi de $18,4 \mathrm{~cm}$. 0 número total de anéis contabilizados do primeiro ao último foi de 106 anéis. Já no segundo grupo (fêmeas), o comprimento traqueal foi de 12,4cm e o número total de anéis, 101.

A luz da traqueia de $B$. variegatus revelou-se ovalada, com um ligeiro achatamento dorso-ventral, semelhante a dos gansos (Santos et al. 2008) e diferente dos humanos (Gamsu \& Webb 1982) e de S. sciureus (Pinheiro et al. 2012) já que a luz variou em sentido lateral.

Segundo Straus \& William (1931) o número de anéis traqueais variou até dentro do mesmo gênero, o que foi observado em $B$. variegatus, já que houve uma pequena diferença nos onze exemplares estudados. Estes mesmos autores afirmaram que no final da traqueia, antes do primeiro brônquio, os anéis podem assumir formas um tanto quanto bizarras, fato também constatado na traqueia da $B$. variegatus uma vez que a porção final revelou sinuosidades.

Destacou-se ainda, durante esta investigação, o fato dos pulmões da preguiça comum, não serem lobados, nos quais pudemos observar apenas o pulmão direito e o pulmão esquerdo, com ausência de fissuras, (Fig.1A e B) ao contrário do pulmão de vários animais como o do quati (Nasua nasua) (Oliveira et al. 2012), da cutia (Penno et al. 2005) e da capivara (Citrângulo et al. 2001), além dos mamíferos em geral (Getty 1986, Dyce et al. 2004).

A literatura é escassa, no que se refere às descrições morfológicas e aos aspectos morfométricos da traqueia de Xenartras. De maneira geral, a traqueia de Bradypus variegatus assemelhou-se estruturalmente aquela encontrada nos outros mamíferos por apresentar os anéis traqueais incompletos (Dyce et al. 2004), a exceção das porções torácicas sinuosas, conforme supra mencionado.

Levando em consideração a dificuldade natural de realizar procedimentos como a IOT (Intubação orotraqueal) nesta espécie, devido à arquitetura natural da traqueia, é comum a ocorrência de quadros moderados a grave de apneia, bradicardia e hipóxia resultantes da hipoventilação (Gilmore et al. 2000). Sendo assim, a escolha de sondas endotraqueais adequadas para uso em Bradypus variegatus, seja para filhotes ou animais adultos, torna-se um desafio para médicos veterinários, devido ao comprimento, largura e sinuosidade própria do órgão. Para abordar situações onde haja a necessidade de rápido restabelecimento da função respiratória, a opção viável é a realização de traqueostomia e colocação de sonda específica, caracterizando um procedimento emergencial para manutenção da ventilação-perfusão pulmonar. Além da dificuldade da realização da IOT, há também falta de disponibilidade de sondas adequadas para esta espécie, portanto, a traqueostomia tornar-se-ia o método alternativo para manter as vias aéreas pérvias, porém, tendo em vista que a região mais indicada para esse acesso seria por meio de incisão na região cervico-caudal, entre a porção caudal da última flexura e a carina traqueal, estando estas situadas já em cavidade torácica, tal acesso seria impossível.

Problemática semelhante foi descrita Brainard e colaboradores (2008) em tamanduás-bandeira (Myrmecophaga tridactyla), uma vez que a morfologia traqueal desta es- 
pécie também é peculiar, conferindo a mesma dificuldade para a realização da IOT nos procedimentos anestésicos, o que os levou a optar pela traqueostomia ao invés da IOT. As sondas de traqueostomia compatíveis para uso nesta espécie são as sondas indicadas para uso humano, preferencialmente de material plástico, flexíveis e adequadas para a utilização em crianças e, os diâmetros correspondentes da sonda variam de acordo com o peso e idade do animal, podendo utilizar nos filhotes as sondas indicadas para pacientes humanos neonatal e para os animais adultos, as de tamanho infantil (Fraga et al. 2009).

\section{CONCLUSÕES}

A traqueia da preguiça comum Bradypus variegatus apresentou três sinuosidades em dois espécimes e duas sinuosidades nas outras nove, independente do sexo e idade dos animais estudados, se mostrando diferente a qualquer outra traqueia animal, e que as alturas e larguras de cada região diferiram entre os exemplares.

Mesmo com das dificuldades anatômicas para se utilizar IOT em B. variegatu e considerando as dificuldades do uso da traqueostomia para a manutenção da função respiratória desta espécie, uma vez que a técnica cirúrgica é complexa e trabalhosa devido à localização indicada para a incisão e alocação da cânula, quando necessário deve-se considerar a opção pela IOT na qual a sonda percorrerá um curto espaço da traqueia quando alojada apenas na região do terço cranial, evitando assim, lesões comuns neste procedimento como estenose, laceração e perfuração do órgão.

Agradecimentos.- Ao Museu Emílio Goeldi pelos animais doados. Esse estudo foi parcialmente financiado pela Fundação de Amparo à Pesquisa do Pará (FAPESPA) e pelo CNPq (Projeto Universal) processo no 476656/2013-3.

\section{REFERÊNCIAS}

Amorin M.J.A.A.L., Miglino M.A., Júnior A.A.A. \& Santos T. C. 2003. Aspectos morfológicos da placenta da preguiça, Bradypus variegatus Shinz,1825. Braz. J. Vet. Res. Anim. Sci. 40(3):217-226.

Brainard B.M., Newton A., Hinshaw K.C. \& Klide A.M. 2008. Tracheostomy in the giant anteater (Myrmecophaga tridactyla). J. Zoo Wildl. Med. 39(4):655-658.

Cáuper G.C.B., Cáuper F.R.M. \& Brito L.L. 2006. Biodiversidade Amazônica: flora amazônica. Centro Cultural dos Povos da Amazônia, Manaus. 162p.

Citrângulo M., Ribeiro A.A.C.M., Moraes P.T.B. \& Machado M.R.F. 2001. Lo- bação e vascularização arterial do pulmão da capivara (Hydrochaeris hydrochaeris). Arq. Ciênc. Vet. Zool. Unipar 4(2):119-127.

Dyce K.M., Sack W.O. \& Wensing C.J.G. 2004. Tratado de Anatomia Veterinária. 3aㅡ ed. Guanabara Koogan, Rio de Janeiro. 813p.

Ettinger S.J. \& Feldman E. 2004. Tratado de Medicina Interna Veterinária. 5a ed. Guanabara Koogan, Rio de Janeiro. 2256p.

Fraga J.C., Souza J.C. \& Kruel J. 2009. Pediatric tracheostomy. J. Pediatr. 85(2):97-103.

Frandson R.D., Wilke W.L. \& Fails A.D. 2005. Anatomia e Fisiologia dos Animais de Fazenda. $6^{\underline{a}}$ ed. Guanabara Koogan, Rio de Janeiro. 279p.

Gamsu G. \& Webb W.R. 1982. Computed tomography of the trachea: normal and abnormal. Am. J. Roentgenol. 139(2):321-326.

Getty R. 1986. Sisson and Grossman's Anatomy of the Domestic Animals. 5th ed. W.B. Saunders, Philadelphia. 1211p.

Gilmore D.P., Da-Costa C.P. \& Duarte D.P.F. 2000. An update on the physiology of two- and three-toed sloths. Braz. J. Med. Biol. Res. 33(2):129-146.

International Committee on Veterinary Gross Anatomical Nomenclature 2012. Nomina Anatômica Veterinária. World Association on Veterinary Anatomist, Knoxville. 160p.

Kuehne B. \& Junqueira L.C.U. 2000. Histologia da traqueia e do pulmão de Siphonops annulatus (Amphibia, Gymnophiona). Revta Bras. Biol. 60:167-172.

König H.E. \& Liebich H.G. 2004. Anatomia dos animais domésticos: texto e atlas colorido. Vol.2. Artmed, Porto Alegre. 400p.

Oliveira C.V., Souza A.F., Santos A.C., Bertassoli B.M., Rosa R.A., Carvalho A.F., Martins J.F.P. \& Mançanares C.A.F. 2012. Estudo morfológico do sistema respiratório de quati (Nasua nasua). Biotemas 25(1):81-92.

Penno A.K., Carvalho M.A.M., Assis-Netto A.C., Azevedo L.M. \& Mello G.W.S. 2005. Lobação, ramificação brônquica e distribuição arterial no pulmão da cutia (Dasyproeta sp., Mammalia - Rodentia). Braz. J. Vet. Res. Anim. Sci. 42(5):331-336.

Pinheiro L.L., Lima A.R., Muniz J.A.P.C., Imbeloni A.A., Fioreto E.T., Fontes R.F., Cabral R. \& Branco E. 2012. Anatomy and morphometric aspects of the trachea of Saimiri sciureus Linnaeus, 1758: knowledge for emergency procedures. Anais Acad. Bras. Ciênc. 84(4):605-608.

Ross M.H \& Pawlina W. 2012. Histologia - Texto e Atlas: em correlação com a biologia celular e molecular. Guanabara Koogan, Rio de Janeiro 1008p.

Santos T.C., Trujillo H.A.G., Alberto M.L.V., Pereira C.C.H., Ferrari C.C., Menconi A. \& Bombonatto P.P. 2008. Aspetos morfológicos e morfométricos da traqueia em gansos domésticos. Biotemas 20(2):91-96.

Straus J.R. \& William L. 1931. The Form of the tracheal cartilages of Primates, with remarks on the supposed taxonomic importance. J. Mammal. 12(3):281-285.

Xavier R.G., Sanches P.R.S., Neto A.V.M., Kuhl G., Vearick S.B. \& Michelon M.D. 2008. Desenvolvimento de uma órtese de Dumon modificada para aplicações traqueais: um estudo experimental em cães. J. Bras. Pneumol. 34(1):21-26. 